\title{
Antibiótico-profilaxia em cirurgia de tumores da cabeça e do pescoço: 24 ou 72 horas?*
}

\author{
Antibiotic prophilaxis in head and neck surgery: 24 or 72 hours?
}

Gyl Henrique Albrecht Ramos,' Benedito Vladecir de Oliveira² e Luis César Bredt ${ }^{3}$

\section{Resumo}

O bjetivos: a antibiótico-profilaxia das cirurgias de tumores malignos da cabeça e do pescoço, pode ser considerada complexa. A pesar de relatos da literatura não demonstrarem diferenças significativas na ocorrência de infecção pós-operatória, antes de mudar-se o tempo de vigência do antibiótico profilático (cefazolina) de 72 para 24 horas instituiu-se um protocolo, de forma prospectiva e randomizada, com a intenção de se avaliar a repercussão desta mudança no ambiente específico do Serviço de Cirurgia da Cabeça e do Pescoço do H ospital Erasto Gaertner.

M aterial e métodos: no período de dezembro de 1993 até agosto/1998, com 93 casos, foi realizado o protocolo direcionado para as pelveglossomandibulectomias (PGM) e para as laringectomias totais (LAR), estratificando-as de forma equival ente, obedecendo-se critérios de elegibilidade e também critérios definindo a existência de infecção.

Resultados: até o momento os casos estão assim distribuídos: 45 PGM e 48 LAR. Dos incluídos no grupo de 24 horas (39 casos), 10\% evoluíram com infecção e dos incluídos no grupo de 72 horas (54 casos), 22\% infectaram.

C onclusão: não se percebeu piora nos índices de ocorrência de infecção pós-operatória no braço 24 horas, sendo indicada a sua utilização. N ovo estudo semelhante está sendo executado, comparando-se agora o tempo de oito horas com o de 24 horas.

Palavras-chave: neoplasias de cabeça e pescoço; infecção da ferida operatória; antibióticoprofilaxia; cefazolina.

*X Jornada de O ncologia do H ospital Erasto Gaertner, outubro de 2000. XV Congresso Brasileiro de Cancerologia, novembro de 2000.

${ }^{1}$ Cirurgião do Serviço de Cirurgia de Cabeça e Pescoço, H ospital Erasto Gaertner, Curitiba - PR. M estre em Cirurgia de C abeça e Pescoço. Especialista em Cirurgia de $\mathrm{C}$ abeça e Pescoço pela Sociedade Brasileira de Cirurgia de $\mathrm{C}$ abeça e Pescoço; Especialista em C ancerologia pela Sociedade Brasileira de Cancerologia. Responsável pela Cirurgia de C abeça e Pescoço do D epartamento de 0 torrinolaringologia da U niversidade Federal do Paraná.

${ }^{2}$ Chefe do Serviço de Cirurgia de Cabeça e Pescoço, H ospital Erasto Gaertner, Curitiba - PR. Especialista em Cirurgia de Cabeça e Pescoço pela Sociedade Brasileira de Cirurgia de Cabeça e Pescoço. Especialista em Cancerologia pela Sociedade Brasileira de Cancerologia. Enviar correspondência para B.V.O. H ospital Erasto Gaertner, Serviço de Cirurgia de Cabeça e Pescoço, Rua Dr. O vande do Amaral 201; 81520-060 Curitiba, PR - Brasil. E-mail: cepep@lpccnet.org.br

${ }^{3} \mathrm{M}$ édico Residente do Hospital Erasto Gaertner, Curitiba - PR.

Recebido em janeiro de 2002. 


\section{Abstract}

0 bjectives: the antibiotic prophylaxis in oncologic head and neck surgery is a complex issue. D espite of literature reports do not show significant differences in the occurrence of wound infection, before changing the time of prophylaxis of 72 hours to 24 hours, a prospective and randomized trial was set up. The trial was conducted in the Erasto Gaertner H ospital - H ead and N eck Surgery D epartment.

M ethods. from D ecember, 1993 to August,1998, 93 patients submitted to total laryngectomy and pelviglossomandibulectomy were included in the protocol, with detailed information about the antibiotic prophylaxis time and the existence of wound infection.

Results. the 24 hour group (39 cases) had a 10\% infection rate, and the 72 hours group had a $22 \%$ infection rate.

Conclusion: there was no significant differences in the infection rate between the groups. A similar trial has been conducted comparing 8 hours versus 24 hours of antibiotic prophylaxis.

Key words head and neck neoplasms, surgical wound infection; antibiotic prophylaxis; cefazolin.

\section{INTRODUÇÃO}

Infecção cirúrgica pós-operatória é uma importante causa de morbidade após cirurgia de cabeça e pescoço, ${ }^{1}$ que pode prolongar a hospitalização, aumentar custos médicos e retardar a cicatrização da ferida operatória. Em pacientes oncológicos, o atraso da cicatrização pode significar uma demora na administração da radioterapia pós-operatória, aumentando o índice de recorrência tumoral.

0 risco de infecção cirúrgica em cabeça e pescoço varia com a característica do procedimento. ${ }^{2} \mathrm{Em}$ feridas limpas, o risco de infecção sem 0 uso de antibiótico peroperatório é menor do que $1 \% \cdot{ }^{4}$ Então, 0 uso rotineiro da antibiótico-profilaxia nestes casos não se mostrou benéfico aos pacientes. ${ }^{2,3}$ Shapiro ${ }^{4}$ também notou que 0 risco de infecção em pacientes submetidos a procedimentos cirúrgicos limpos em cabeça e pescoço era muito baixo $(<1 \%)$ e que a administração peroperatória de antibióticos nestes pacientes não é indicada. Weber e C allender ${ }^{2}$ concluíram numa análise custobenefício que a administração de antibióticos no peroperatório neste mesmo grupo de pacientes não é indicada.

Em contraste, procedimentos potencialmente contaminados (feridas inicialmente estéreis, mas com a quebra de uma barreira mucosa ou abertura de uma víscera oca durante a cirurgia) ${ }^{2}$ demonstram um risco significativo de infecção. Seagle $e^{5}$ demonstrou em um estudo que $48 \%$ dos pacientes que não receberam antibiótico peroperatório evoluíram com infecção. U ma revisão de nove estudos demonstrou taxas de infecção em vários tipos de cirurgia de cabeça e pescoço entre $18 \%$ e $87 \%$, comparados com taxas entre $8 \%$ e $38 \%$ quando com o uso de antibiótico-profilaxia. ${ }^{4}$ Sem 0 uso de antibióticos peroperatório, cirurgias contaminadas de cabeça e pescoço apresentam um índice relativamente alto de infecção. Além do mais, a infecção pode eventualmente ocorrer mesmo em pacientes que recebem tal terapia. ${ }^{4}$

Apesar de relatos da literatura não demonstrarem diferenças significativas na ocorrência de infecção pós-operatória quanto ao tempo de antibiótico-profilaxia, antes de mudar-se o tempo de vigência do antibiótico profilático (cefazolina) de 72 para 24 horas instituiu-se um protocolo com a intenção de se avaliar a repercussão desta mudança no ambiente específico do Serviço de Cirurgia da Cabeça e do Pescoço do H ospital Erasto Gaertner.

\section{MATERIAIS E MÉTODOS}

De dezembro de 1993 a agosto de 1998, por um período total de 56 meses, 93 pacientes foram avaliados de maneira prospectiva, randomizada, duplo-cego, conforme protocolo direcionado para pacientes submetidos à 
laringectomia total (LGT) ou pelviglossomandibulectomia (PGM). O s pacientes participantes do estudo só foram considerados elegíveis após a adequada compreensão do procedimento a ser realizado com assinatura do termo de consentimento pós-informado. 0 estudo foi realizado no H ospital Erasto Gaertner em Curitiba (PR) pelo Serviço de Cirurgia de Cabeça e Pescoço.

0 protocolo incluía informações de caráter demográfico, parâmetros nutricionais, características tumorais, parâmetros cirúrgicos, dados de transfusão sangüínea, tempo de antibiótico-profilaxia e presença ou não de infecção pós-operatória (a distância, sistêmica ou local).

Foram analisados apenas os dados referentes à ocorrência de infecção de ferida operatória (IFO) e o tempo de antibióticoprofilaxia, a qual consistia na administração de cefazolina 1000 mg a cada 8 horas. N ossa definição de IFO inclui infecção incisional ou profunda da ferida cirúrgica, baseada nos parâmetros clínicos, sem a obrigatoriedade de uma cultura positiva.

Foram correlacionados os índices de IFO com o tempo de antibiótico-profilaxia: 24 ou 72 horas, comparando-se as porcentagens por análise estatística pertinente (qui-quadrado), sob a supervisão do Serviço de Estatística do $\mathrm{H}$ ospital Erasto Gaertner.

\section{RESULTADOS}

$\mathrm{Na}$ análise do procedimento cirúrgico realizado, 48 pacientes foram submetidos à laringectomia total (LGT) e 45 à pelviglossomandibulectomia (PGM ). 0 grupo que recebeu 24 horas de cefazolina profilática consistiu de 39 pacientes, enquanto o grupo de 72 horas totalizou 54 pacientes, sendo que a taxa global de infecção, independente do tempo de antibiótico-profilaxia, foi de $17 \%$.

$\mathrm{Na}$ análise específica de cada grupo, foram obtidos os seguintes resultados: 24 horas de antibiótico-profilaxia apresentou $10 \%$ de incidência de IFO; 0 de 72 horas de antibiótico-profilaxia, 22\%. N ão foi verificada diferença estatística na incidência de IFO entre os dois grupos estudados, com o valor de $p=0,12$.

\section{DISCUSSÃO}

D entre os antibióticos citados na literatura utilizadas em pacientes submetidos à cirurgia de cabeça e pescoço, incluem-se cefazolina, cefotaxima, ceftriaxone, cefamandole, cefoperazona, clindamicina, moxalactam, gentamicina + clindamicina, cefazolina + metronidazol, ticarcilina + ácido clavulânico, e ampicilina + sulbactam. ${ }^{5} \mathrm{O}$ s resultados obtidos com essas drogas enfatizam a importância de selecionar o tratamento que seja efetivo tanto para microorganismos aeróbios como para anaeróbios, bem como gram-negativos e gram-positivos. Callender ${ }^{3}$ ressaltou, num trabalho prospectivo comparando o uso de sulbactam/ampicilina com clindamicina, a importância da cobertura de microorganismos gram-negativos em pacientes submetidos à cirurgia oncológica de cabeça e pescoço.

Antibióticos beta-lactâmicos combi-nados com inibidores da beta-lactamase (ticarcilina/ clavulanato e ampicilina/ sulbactam), derivados das cefalosporinas (cefotaxima e cefazolina), e gentamicina possuem uma efetiva ação contra os principais microorganismos. A penas a gentamicina possui uma boa atividade contra o Pseudomonas aeruginosa. ${ }^{6}$

Osmicroorganismos endógenos envolvidos em procedimentos oncológicos de cabeça e pescoço incluem a flora da pele, predominantemente $S$ aureus, Staphilococcus epidermidis, e streptococcus beta-hemolítico, e os microorganismos comensais do trato aerodigestivo alto. $\mathrm{N}$ as secreções da orofaringe, os organismos anaeróbios são dez vezes mais comuns que os aeróbios, com uma quantidade variando de $10^{8} / \mathrm{ml}$ a $10^{9} / \mathrm{ml}$. Estes organismos, que colonizam a língua, dentes e fissuras dentárias, são esfoliados na saliva. Entre os organismos anaeróbios, Bacterioides e Fusobacterium são encontrados em grandes concentrações nas fissuras dentárias. ${ }^{2}$ A Tabela 1 demonstra os patógenos mais comumente associados à infecção da ferida operatória em cabeça e pescoço segundo G erard e M eunier.? 
Tabela 1. Patógenos mais comumente envolvidos em infecção da ferida operatória em cabeça e pescoço.

\begin{tabular}{|c|c|c|}
\hline Grampositiwo Aerobios & Gramrlegat wo Aerobios & A Araeróbios \\
\hline $\begin{array}{l}\text { Staphybococcu aure us } \\
\text { Staphybooccus epidermid s } \\
\text { Stre ptococcus species } \\
\text { Cory nebacte riums peries }\end{array}$ & 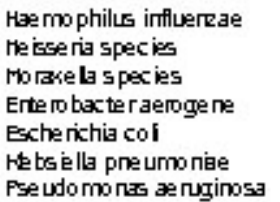 & $\begin{array}{l}\text { Eactioides species } \\
\text { Fuso baxte rum speces } \\
\text { Peptostre pbcocus species }\end{array}$ \\
\hline
\end{tabular}

A cefazolina é uma cefalosporina de primeira geração semi-sintética quevem sendo usada extensivamente para o tratamento peroperatório em cirurgia de cabeça e pescoço. ${ }^{5}$ Possui uma ação contra organismos aeróbios, ${ }^{1}$ contudo é relativamente inefetiva contra bactérias anaeróbias. U m estudo comparando cefazolina com placebo em 50 pacientes, mostrou que 0 uso a curto prazo de cefazolina profilática mostrou-se importante na redução de infecção pósoperatória em cabeça e pescoço. ${ }^{8}$ Em 1979 , Becker e Parrel ${ }^{14}$ relataram os resultados de um estudo randomizado, duplo-cego, também comparando a cefazolina com placebo em 55 pacientes submetidos a procedimentos limpocontaminados em cabeça e pescoço. A taxa de infecção nos pacientes que receberam cefazolina foi de $38 \%$, comparado com $87 \%$ naqueles que receberam placebo.

Três grandes estudos abordando procedimentos oncológicos em cabeça e pescoço demonstram que os regimes combinados com cobertura para aeróbios e anaeróbios foram superiores à cefazolina para prevenção de infecções. Num estudo prospectivo, a cefazolina foi combinada com metronidazol, um agente altamente específico contra anaeróbios. A taxa de infecção entre 172 pacientes que receberam apenas cefazolina como profilaxia, foi de $18,6 \%$, comparado a 9,5\% em 158 pacientes tratados com a combinação. ${ }^{5} 0$ utro estudo prospectivo realizado por Brand e colaboradores mostrou taxas de infecção pós-operatória de $27 \%$ entre 83 pacientes que receberam um ou cinco dias de cefazolina, e apenas $7 \%$ em pacientes que receberam gentamicina+clindamicina. ${ }^{2}$ Johnson e colaboradores relataram taxas de infecção pós-operatória de $25,5 \%$ com monoterapia de cefazolina, e 5,4\% com a combinação de gentamicina e clindamicina em 107 pacientes. ${ }^{9}$ Em contrapartida, um estudo prospectivo randomizado, duplo-cego, realizado por Juan, ${ }^{10}$ em 159 pacientes, comparando amoxacilina-clavulanato, clindamicina e cefazolina apresentou índices de infecção de $22,8 \%, 21,2 \%$ e $26 \%$ respectivamente, não se verificando diferença estatística. Em outro estudo, Johnson et al ${ }^{15}$ randomizou 118 pacientes para receber moxalactan, uma cefalosporina de terceira geração, ou cefazolina, cada uma em dose de $2 \mathrm{~g}$ a cada 8 horas por um total de 4 doses. A infecção da ferida operatória ocorreu em 7 pacientes (6\%), 5 no braço da cefazolina e dois no braço do moxalactan, uma diferença que não foi significativa. ${ }^{2}$

Além de Robins et al, ${ }^{5}$ Strauss $^{11}$ comprovou a eficácia da associação cefazolina + metronidazol em cirurgias oncológicas de cabeça e pescoço, com uma taxa de infecção de $28,1 \%$, obtendo resultados equival entes a outros esquemas de antibiótico citados na literatura. No Serviço de C irurgia de C abeça e Pescoço do Hospital Erasto Gaertner, a associação do metronidazol à cefazolina profilática é realizada apenas em casos selecionados: pós-radioterapia ou em presença de extensa necrose tumoral ou linfonodal detectada no pré ou intra-operatório.

As informações disponíveis na literatura, referentes ao tempo ideal de antibióticoprofilaxia em cirurgia oncológica de cabeça e pescoço, não nos permitem fazer afirmações inequívocas sobre este tema. Bhathena ${ }^{12}$ comparou pacientes submetidos a ressecções oncológicas com rotação de retal ho em cabeça e pescoço, e não verificou diferença na incidência de infecção pós-operatória entre o grupo que recebeu um dia de cefaperazona profilática contra o grupo que recebeu cefotaxima por cinco dias. O utro estudo randomizado comparando a eficácia da clindamicina-cefocinide administradas por 24 horas $\times 72$ horas revelou resultados equivalentes na incidência de infecção pósoperatória. ${ }^{13}$

Ao contrário da maioria dos trabalhos citados, este estudo avaliou de maneira prospectiva uma amostragem de pacientes com características semelhantes, objetivando uma maior confiabilidade às informações obtidas. $\mathrm{N}$ ão se verificou uma diferença estatisticamente significativa nos índices de 
IFO entre os grupos que receberam 24 horas $(10 \%)$ e 72 horas $(22 \%)$ de cefazolina profilática $(p=0,12)$, reforçando assim a necessidade da continuação dos estudos relacionando o tempo de antibiótico-profilaxia e a incidência de infecção da ferida operatória em cirurgia oncológica de cabeça e pescoço.

\section{REFERÊNCIAS BIBLIOGRÁFICAS}

1. Weber RS, H ankins PP, Rosenbaum B, et al. $N$ onwound infections following head and neck oncologic surgery. Laryngoscope1993;103:22-7.

2. Weber RS, Callender D L. Antibiotic prophylaxisin clean-contaminated head and neck oncologic surgery. Ann 0 tol R hinol Laryngol 1992:101:16-20.

3. Callender D L. Antibiotic prophylaxisin head and neck oncologic surgery: the role of gramnegative coverage. Int J Antimicrob Agents 1999;(12Suppl1):S21-5.

4. Shapiro M . Prophylaxisin otolaryngologic surgery and neurosurgery: a critical review. Rev Infect D is 1991;13(Suppl 10):S858- 68.

5. RobbinsKT, ByersRM, ColeR, et al. Wound prophylaxis with metronidazole in head and neck surgical oncology. Laryngoscope 1988;98:803-6.

6. Weber RS. Wound infection in head and neck surgery: implications for perioperativeantibiotic treatment. Ear NoseT hroatJ 1997;76(11):790-1.

7. Gerard M, M eunier F. Antimicrobial prophylaxisfor major head and neck surgery in cancer patients. Antimicrob Agents Chemother 1988;32:1557-9.
8. Seagle M B, D uberstein LE, Gross CW, et al. Efficacy of cefazolin as a prophylactic antibiotic in head and neck surgery. Trans Am Acad O phthalmol 0 tol 1978; 86:568-72.

9. Johnson JT, Kachman $K$, Wagner $R L$, M yers EN . Comparison of ampicillin/sulbactam versusclindamycin in the prevention of infection in patientsundergoing head and neck surgery. $\mathrm{H}$ ead N eck 1997;19(5):367-71.

10. Rodrigo JP, Alvarez JC, Gomez JR, Suarez C, Fernandez JA, M artinez JA. Comparison of threeprophylactic antibiotic regimensin cleancontaminatehead and neck surgery. H ead N eck 1997;19(3):188-93.

11. Strauss M, Saccogna PW, Allphin AL. Cephazolin and metronidazol prophylaxis in head and neck surgery. J Laryngol 0 tol 1997;111(7):631-4.

12. Bhathena H M , Kavarana N M . Prophylactic antibioticsadministration head and neck surgery with major flap reconstruction: 1-day cefoperazoneversus5-day cefotaxime.ActaChir Plast 1998;40(2):36-40.

13. Righi M , M anfredi R, Farneti G, Pasquini E, Cenacchi V. Short-term versus long-term antimicrobial prophilaxis in oncologic head and neck surgery. Head N eck 1996;18(5):399404.

14. Becker GD , Parrel GJ . C efazolin prophylaxis in head and neck surgery. Ann 0 tol Rhinol Laryngol 1979;88:183-6.

15. Johnson JT, Yu VL, M yers EM, Wagner RL, Sigler BA. C efazolin vs moxal actan? A double blind randomized trial of cephalosporins in head and neck surgery. Arch 0 tolaryngol $\mathrm{H}$ ead N eck Surg 1986;112:151-3. 